Research Paper

\title{
Efficient Inhibition of Ovarian Cancer by Gelonin Toxin Gene Delivered by Biodegradable Cationic Heparin-polyethyleneimine Nanogels
}

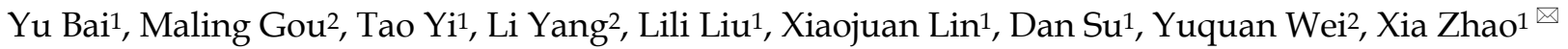 \\ 1. Department of Gynecology and Obstetrics, Key Laboratory of Obstetric and Gynecologic and Pediatric Diseases and Birth Defects of \\ Ministry of Education, West China Second Hospital, Sichuan University, Chengdu, Sichuan 610041, P.R. China \\ 2. State Key Laboratory of Biotherapy and Cancer Center, West China Hospital, Sichuan University, Chengdu, Sichuan 610041, P.R. China
}

\begin{abstract}
$\bowtie$ Corresponding author: Xia Zhao, xia-zhao@126.com; Tel:+86-28-85502822; Fax: +86-28-85502822
(c) 2015 Ivyspring International Publisher. Reproduction is permitted for personal, noncommercial use, provided that the article is in whole, unmodified, and properly cited. See http://ivyspring.com/terms for terms and conditions.
\end{abstract}

Received: 2014.10.28; Accepted: 2015.02.25; Published: 2015.05.08

\begin{abstract}
The use of toxins for cancer therapy has great promise. Gelonin, a potent plant toxin, causes cell death by inactivating the $60 \mathrm{~S}$ ribosomal subunit. Recently, we developed a novel gene delivery system using biodegradable cationic heparin-polyethyleneimine (HPEI) nanogels. In the current study, the antitumor activity of a recombinant plasmid expressing gelonin (pGelonin) on human ovarian cancer was assessed. The application of HPEI nanogels, was also evaluated. Gelonin-cDNA was cloned into the PVAX1 plasmid vector and transfected into SKOV3 human ovarian cancer cells using biodegradable cationic HPEl nanogels. The expression of gelonin in vitro and in vivo was confirmed using RT-PCR and western blot analysis. Cell viability and apoptosis were examined using an MTT assay and flow cytometric analysis. For the in vivo study, an SKOV3 intraperitoneal ovarian carcinomatosis model was established, and nude mice were randomly assigned into four groups receiving i.p. administration of pGelonin/HPEl complexes, pVAX/HPEl complexes, HPEI alone and $5 \%$ glucose solution. The tumor weight was monitored, and a TUNEL assay and Ki-67 immunohistochemistry were performed to evaluate apoptosis and cell proliferation in the tumor tissue sections, respectively. Gelonin was efficiently expressed in SKOV3 cancer cells in vitro and in vivo using pGelonin incorporated with HPEI nanogels. The pGelonin/HPEl complexes inhibited cell viability and induced apoptosis in the cell culture. Treatment for intraperitoneal carcinomatosis with pGelonin/HPEl complexes reduced the tumor weight by $\sim 58.55 \%$ compared to the control groups $(P<0.05)$. The antitumor effect was accompanied by increased apoptosis and reduced cell proliferation $(P<0.05)$. No significant side effects were observed with i.p. administration of the pGelonin/HPEl complexes. Our data indicate that HPEI nanogel-delivered pGelonin may have promising applications against human ovarian cancer.
\end{abstract}

Key words: gene therapy, ovarian cancer, gelonin toxin, cationic nanogels, heparin-polyethyleneimine (HPEI)

\section{Introduction}

Ovarian cancer is the most lethal gynecologic malignancy with an estimated 22,280 new cases and 15,500 deaths in 2012 (1). Currently, the two major obstacles in treating this life threatening disease are the lack of effective early detection and the emergence of increased drug resistance. Although patients receiving standard therapy, including surgical cytoreduction and platinum-based combination chemo- therapies, may have an initial favorable response, most cases will relapse within 5 years. Consequently, there is an urgent need for novel treatments for this deadly disease.

Gelonin, a 29-kDa single-chain glycoprotein, belongs to a class of potent ribosomal-inactivating plant toxins. By dissecting rRNA adenine $\mathrm{N}$-glycoside bonds in a sequence-specific fashion, gelonin irre- 
versibly inactivates the 605 ribosomal subunit. Different from dual-chain toxins, such as ricin and abrin, gelonin lacks a carbohydrate-binding domain for binding to cells. Consequently, gelonin is relatively nontoxic to intact cells, but highly potent in inhibiting protein synthesis after it gains entry into cells (2). In addition, gelonin does not cause capillary leak syndrome or hepatotoxicity $(3,4)$, and it may be more appropriate for therapeutic applications compared to other toxins. Previous studies utilized gelonin as a cytotoxic reagent chemically conjugated with monoclonal antibodies, hormone or growth factor ligands in the cancer therapeutic field (5). Despite significant cytotoxic activities of these agents, obstacles, such as immunogenicity, short serum half-life, degradation, difficulties of refinement and high cost, have limited their success.

Gene therapy has been extensively studied for ameliorating or curing a variety of diseases by inducing therapeutic nucleic acids into cells. It is an appealing modality for ovarian cancer because the disease tends to remain localized within the peritoneal cavity, creating a rationale for locoregional delivery (6). In addition to the selection of therapeutic genes, the success of gene therapy is largely dependent on the gene delivery system.

Currently, there are two major approaches for gene transfer: using viral and non-viral vectors. Non-viral vectors have gained importance in recent years because of their relative safety, low immunogenicity and toxicity, ease of use and widespread production compared to viral vectors. Among the total non-viral vectors, polyethyleneimine (PEI) is one of the most effective gene transfection agents $(7,8)$. Currently, the commercially available PEI25K (25,000 $\mathrm{g} / \mathrm{mol}$ ) is considered the gold standard for polymer or surfactant-based gene carriers because of its high transfection efficiency. However, PEI is not biodegradable and has its limitations in that both the efficiency and toxicity are strongly correlated with its molecular weight $(9,10)$ with high efficiency accompanied by serious cytotoxicity. To overcome this issue, PEI has undergone several modifications. In our previous study, biodegradable cationic HPEI nanogels were generated by chemically conjugating PEI with heparin. Furthermore, HPEI nanogels were used to effectively deliver plasmid DNA into several cancer cells in our laboratory, with the plasmid/HPEI complexes exhibiting positive antitumor efficiency in different animal models (11-14).

In the current study, we constructed a plasmid vector, which successfully expressed the gelonin toxin, and introduced it into SKOV3 ovarian cancer cells using biodegradable cationic HPEI nanogels. We demonstrate that HPEI nanogels effectively deliver plasmid DNA expressing gelonin toxin to human ovarian cancer cells, resulting in the suppression of cell growth in vitro and significant inhibition of tumor growth in nude mice following i.p. administration.

\section{Materials and Methods}

\section{Cell lines}

The human ovarian cancer cell line, SKOV3, was obtained from the American Type Culture Collection (ATCC, Manassas, VA) and maintained in DMEM (Gibco BRL, Grand Island, N.Y.) supplemented with $10 \%$ fetal calf serum (Gibco, Auckland, N.Z.), $2 \mathrm{mM}$ L-glutamine, $100 \mathrm{U} / \mathrm{ml}$ penicillin and $100 \mathrm{mg} / \mathrm{ml}$ streptomycin. The cells were incubated at $37^{\circ} \mathrm{C}$ in a humidified atmosphere of 5\% $\mathrm{CO}_{2}: 95 \%$ air in an incubator and passaged every 4 days at a split ratio of 1:3 using trypsin.

\section{Plasmid construction and HPEI preparation}

To construct a recombinant plasmid expressing gelonin (pGelonin), a 780-bp cDNA encoding gelonin was synthesized according to the nucleotide sequences available from the National Center for Biotechnology Information (NCBI) database and subcloned into the expression vector pVAX1 (Invitrogen, Sweden). The gelonin cDNA fragment was amplified by PCR with the following two primers: 5'-TATGGATCCACTATGGGCCTGGACACCGTGA GCTT-3' (forward primer) and 5'-TACCTCGAGCTA TTTAGGATCTTTATCGACGAAC-3' (reverse primer). The PCR cycling conditions were as follows: denaturation at $94^{\circ} \mathrm{C}$ for $2 \mathrm{~min}$, amplification for 30 cycles at $94^{\circ} \mathrm{C}$ for $30 \mathrm{~s}$, annealing at $60^{\circ} \mathrm{C}$ for $30 \mathrm{~s}$, and extension at $54.3^{\circ} \mathrm{C}$ for $50 \mathrm{~s}$, followed by a terminal elongation step at $72^{\circ} \mathrm{C}$ for $10 \mathrm{~min}$, using Ex Taq polymerase (Takara Shuzo, Kyoto, Japan). The (BamHI+ XhoI)-digested PCR product was ligated to (BamHI+ XhoI)-digested pVAX1. pGelonin was confirmed by restriction digestion and DNA sequencing. As a control, pVAX1 plasmid without the gelonin cDNA fragment was used as an empty vector. Plasmid DNA was amplified in DH5a Escherichia coli and purified using EndoFree Plasmid Giga kits (Qiagen, Chatsworth, CA). The DNA was stored at $-20^{\circ} \mathrm{C}$ for future use at a concentration of $2 \mathrm{mg} / \mathrm{ml}$.

Cell transfection was performed using biodegradable HPEI (heparin-polyethyleneimine) nanogels generated at the State Key Laboratory of Biotherapy, as previously described (11). HPEI nanogels were prepared using amide bond formation through the covalent interaction of the amine groups of the branched PEI with the carboxyl groups of heparin. Briefly, $50 \mathrm{mg}$ heparin $\left(\mathrm{M}_{\mathrm{w}}=4000-6000\right)$ was dissolved in $100 \mathrm{ml} 0.05 \mathrm{M}$ 2-(N-morpholino)ethanesulfonic acid (MES) buffer solution. Subsequently, $20 \mathrm{mg}$ 1-ethyl- 
3-(3-dimethylaminopropyl)carbodiimide (EDC) and $30 \mathrm{mg}$ N-hydroxysuccinimide (NHS) were added into the solution to activate the carboxylic acid groups of heparin. The solution was placed at room temperature for $2 \mathrm{~h}$ and then dropped into $20 \mathrm{ml}$ PEI2K solution $(7.5 \mathrm{mg} / \mathrm{ml})$ with consistent stirring. The reaction was performed at room temperature overnight. The resultant HPEI nanogels were dialyzed in distilled water for $72 \mathrm{~h}$ and filtered with a syringe filter. The HPEI nanogels were stored at $4^{\circ} \mathrm{C}$ before use, at a concentration of $1 \mathrm{mg} / \mathrm{ml}$.

\section{Cell Transfection}

For in vitro transfection, SKOV3 ovarian cancer cells $\left(1.5 \times 10^{5}\right)$ were seeded in each well of a 6-well plate in triplicate and cultured for $24 \mathrm{~h}$ to $50-60 \%$ confluence. A total of $10 \mu \mathrm{g}$ HPEI and $2 \mu \mathrm{g}$ of plasmid DNA were prepared in $1 \mathrm{ml}$ of DMEM medium without antibiotics and serum. In addition, medium alone and $10 \mu \mathrm{g}$ HPEI alone were also used as controls. The cells were incubated with pGelonin/HPEI complexes, pVAX/HPEI complexes, HPEI nanogels or medium alone. After $6 \mathrm{~h}$, the medium was replaced with $2 \mathrm{ml}$ of DMEM supplemented with fetal calf serum and incubated for an additional $48 \mathrm{~h}$. Then, the cells were harvested for further study.

To determine the optimal plasmid:HPEI ratio $(\mu \mathrm{g} / \mu \mathrm{g})$ for efficient gene delivery, pGFP, the green fluorescent protein (GFP) plasmid, was used as a reporter gene. The cells were transfected with various pGFP:HPEI ratios and observed under a fluorescence microscope $48 \mathrm{~h}$ after transfection.

\section{Western Blot Analysis}

Western blot analysis was performed to examine the expression of gelonin in SKOV3 cells or tumor tissues. The cells were scraped from 6-well dishes, washed twice in PBS, and resuspended in RIPA lysis buffer containing $50 \mathrm{mM}$ Tris- $\mathrm{HCl}(\mathrm{pH} \mathrm{7.4}), 0.25 \%$ sodium deoxycholate, $150 \mathrm{mM} \mathrm{NaCl}, 0.1 \%$ SDS, $1 \%$ NP-40, $1 \mathrm{mM}$ EDTA, $1 \mathrm{mM} \mathrm{NaF}$, and $1 \mathrm{mM} \mathrm{Na}_{3} \mathrm{VO}_{4}$, supplemented with proteinase inhibitor $(1 \mathrm{mM}$ cocktail plus $1 \mathrm{mM}$ PMSF). Tumor tissues were pulverized with a mortar and pestle in liquid nitrogen and then lysed in RIPA lysis buffer. The extracts were centrifuged at 15,000 at $4^{\circ} \mathrm{C}$ for $30 \mathrm{~min}$, and the protein concentrations of the supernatants were determined using a Bio-Rad protein assay kit (Bio-Rad Laboratories, Hercules, CA). An equal amount of protein was separated by $12 \%$ SDS-PAGE and transferred onto polyvinylidene difluoride (PVDF) membranes (Millipore, Bedford, MA). The membranes were blocked with $5 \%$ nonfat milk for $1 \mathrm{~h}$ at room temperature and then incubated with rabbit monoclonal antibodies against gelonin (1:200 dilution; a gift from Prof. Li
Yang, Sichuan University, Chengdu, China) at $4^{\circ} \mathrm{C}$ overnight, followed by incubation with a horseradish peroxidase-conjugated anti-rabbit secondary antibody (1:5000; Abcam, Cambridge, MA). The bands were visualized using an enhanced chemiluminescence detection kit (Thermo Scientific). For protein loading analysis, a monoclonal GAPDH antibody $(1: 10,000)$ was used. The bands were scanned and quantified using the Quantity One 4.52 software (Bio-Rad Ltd., USA).

\section{Cytotoxicity assay in vitro}

MTT assay was used to measure the activity of pGelonin on SKOV3 ovarian cancer cells in vitro. Cells $\left(3 \times 10^{3}\right)$ were cultured in 96-plates in $0.2 \mathrm{ml}$ of DMEM medium. After overnight incubation, the pGelonin/HPEI complexes were added to transfect the cells in triplicate. The pVAX/HPEI complexes, HPEI nanogels or medium alone were used as controls. The cells were incubated for $48 \mathrm{~h}$, and then $10 \mu \mathrm{l}$ of MTT ( $5 \mathrm{mg} / \mathrm{ml}$ in PBS; Sigma Chemical Co.) was added to each well and cultured in the dark for an additional $4 \mathrm{~h}$. The supernatant was replaced with 100 $\mu \mathrm{l}$ of DMSO/well. The plates were mixed on a shaker for $15 \mathrm{~min}$ and the absorbance at $490 \mathrm{~nm}$ was determined with a microplate reader. The experiments were performed in triplicate. The reduction of cell proliferation (\%) was determined using the following formula: cell inhibition $(\%)=\left(1-\mathrm{A}_{490}\right.$ of treated cells $/ \mathrm{A}_{490}$ of control cells) $\times 100 \%$.

\section{Flow Cytometric Analysis}

Cellular apoptosis was quantified by flow cytometric analysis using PI staining methods. Briefly, $48 \mathrm{~h}$ after pGelonin/HPEI incubation, both floating and attached cells were collected and washed twice with cold PBS. The cells were resuspended in prediluted binding buffer and propidium iodide $(1 \mu \mathrm{g} / \mathrm{ml})$ was then added. The samples were immediately analyzed on an EPICS ELITE ESP flow cytometer (Beckman Coulter, Hialeah, Fla., USA).

\section{Tumor growth and treatment in vivo}

All animal protocols were approved by the Institutional Animal Care and Use Committee of Sichuan University and were in compliance with standard guidelines. Female athymic nude mice (BALB/c, 6-8 weeks old, non-fertile, 18-20 g each) were used to establish the intraperitoneal carcinomatosis model according to a previous study in the State Key Laboratory of Biotherapy (15). Briefly, $100 \mu \mathrm{l}$ of cell suspension containing $5 \times 10^{6}$ SKOV3 cells were injected s.c. into the backs of four nude mice. When the tumor diameters were approximately $1 \mathrm{~cm}$, the tumors were harvested and ground into tiny particles with a diameter $\leq 1 \mathrm{~mm}$. The tumor particles were 
resuspended with DMEM medium to reach a final volume of $10 \mathrm{ml}$, and 20 nude mice were inoculated i.p. with $0.5 \mathrm{ml}$ of the above suspension. Intraperitoneal administrations were initiated 5 days after inoculation. The mice were separated randomly into four groups of five animals. The groups were as follows: (a) untreated, $100 \mu \mathrm{l}$ of $5 \%$ glucose solution; (b) HPEI, $50 \mu \mathrm{g}$ of HPEI nanogels in $100 \mu \mathrm{l}$ of $5 \%$ glucose solution; (c) pVAX/HPEI, $5 \mu \mathrm{g}$ of pVAX / $50 \mu \mathrm{g}$ of HPEI nanogels in $100 \mu \mathrm{l}$ of $5 \%$ glucose solution; and (d) pGelonin/HPEI, $5 \mu \mathrm{g}$ of pGelonin /50 $\mu \mathrm{g}$ of HPEI nanogels in $100 \mu \mathrm{l}$ of $5 \%$ glucose solution. The DNA/HPEI complexes were prepared and incubated at room temperature for $30 \mathrm{~min}$ before administration. The mice were sacrificed after 10 treatments (once every three days), and the intraperitoneal tumors were resected and weighted.

\section{Immunohistochemical and immunofluores- cence analysis}

Intraperitoneal tumor tissues were collected and either embedded in paraffin or in OCT media for snap freezing. Sections (3-5 $\mu \mathrm{m})$ of formalin-fixed, paraffin-embedded specimens were deparaffinized, dehydrated and then processed with high-temperature antigen retrieval. Immunostaining for gelonin and Ki-67 was performed with rabbit anti-gelonin antibody (diluted 1:200; a gift from Prof. Li Yang, Sichuan University, Chengdu, China), and mouse anti-human Ki-67 antibody (diluted 1:100; Santa Cruz Biotechnology, Santa Cruz, CA), respectively. All specimens were evaluated using an Olympus BX600 microscope and Spot Flex camera. To quantify the proliferation index, the percentage of $\mathrm{Ki}$-67-positive cells was counted in 10 random $0.011-\mathrm{mm}^{2}$ fields at $\times 400$ magnification.

TUNEL assay using a DeadEndTM Fluorometric TUNEL System (Promega, Madison, WI) was performed to detect apoptotic cells in the tumor tissues according the manufacturer's protocol. Cell nuclei with bright green fluorescent staining were defined as TUNEL-positive nuclei. TUNEL-positive nuclei were observed with a fluorescence microscope. The number of TUNEL-positive nuclei was counted in 10 random $0.011-\mathrm{mm}^{2}$ fields at $\times 400$ magnification.

\section{Evaluation of Toxicity}

To evaluate potential side effects and toxicity in the pGelonin-treated mice, relevant indices, such as weight loss, diarrhea, skin ulcerations, ruffled fur or toxic deaths were monitored. Tissues from the heart, liver, spleen, lung and kidney were fixed in $4 \%$ paraformaldehyde solution and embedded in paraffin. Sections of 3 to $5 \mu \mathrm{m}$ were stained with hematoxylin and eosin (H\&E) and observed by two pathologists in a blinded manner.

\section{Statistical analysis}

Data were recorded as the means \pm SD. Comparisons among different groups were performed with one-way ANOVA using the SPSS software. A $p$ value less than 0.05 was considered statistically significant.

\section{Results}

\section{Plasmid construction}

A 780-bp cDNA encoding gelonin was PCR amplified. Agarose gel electrophoresis revealed one band approximately 780-bp in size as expected. The purified PCR products were cloned into the $\mathrm{pVAX} 1$ vector with BamHI and XhoI restriction endonucleases. The identity of the recombinant plasmid pGelonin was verified by PCR, double digestion and DNA sequencing (data not shown).

\section{Preparation and characterization of HPEI}

As shown in Fig. 1A, biodegradable cationic HPEI nanogels were synthesized using amide bond formation of the branched PEI and heparin in the presence of EDC/NHS. The HPEI nanogels were then used to transfect pGFP into SKOV3 cells. At $48 \mathrm{~h}$ after transfection, the cells were observed under a fluorescence microscope. Maximum expression was observed when $2 \mu \mathrm{g}$ plasmid:10 $\mu \mathrm{g}$ HPEI was used (Fig. 1B).

\section{Expression of Gelonin in vitro}

Semi-quantitative RT-PCR and western blot analysis were used to measure the levels of gelonin mRNA and protein in SKOV3 ovarian cancer cells transfected with pGelonin/HPEI complexes. SKOV3 cells were seeded in a six-well plate and transiently transfected with pGelonin/HPEI complexes, pVAX/HPEI complexes, HPEI nanogels or medium alone. RT-PCR and western blot analysis were performed $48 \mathrm{~h}$ after incubation. As shown in Fig. 1C, the data obtained from the RT-PCR and western blot analysis were consistent, indicating successful expression of gelonin in SKOV3 cells transfected with pGelonin/HPEI complexes, whereas there was no expression of gelonin in SKOV3 cells from the pVAX/HPEI complexes, HPEI nanogels or medium alone groups.

\section{Suppression of cell viability and induction of cell apoptosis by pGelonin/HPEI complexes in vitro}

SKOV3 ovarian cancer cells were assayed for cell viability after treatment with the pGelonin/HPEI complexes. The MTT assay revealed that pGelon- 
in/HPEI complexes significantly reduced cell viability compared to the three control groups (Fig. 2A., $\mathrm{P}<0.05)$.

Cell apoptosis was evaluated using flow cytometric analysis. As shown in Fig. 2B, pGelonin/HPEI complexes induced more apoptosis than the control agents. The percentage of apoptotic cells significantly increased in the pGelonin/HPEI group (50.5\%), compared to the pVAX/HPEI (20.1\% ), HPEI $(14.8 \%)$ or medium alone $(5.5 \%)$ groups.

\section{Expression of gelonin in vivo}

To examine the expression of gelonin in vivo, an intraperitoneal ovarian carcinomatosis model was established and nude mice were treated with pGelonin/HPEI complexes, pVAX/HPEI complexes, HPEI nanogels or medium alone. At 3 days after the final treatment, intraperitoneal tumors were collected for RT-PCR, western blot analysis and immunostaining. The expression of gelonin mRNA and protein were detected in tumor tissues from the pGelonin/HPEI complexes-treated group, whereas the pVAX/HPEI complexes, HPEI nanogels or medium groups had no expression of gelonin (Fig. 3A). Furthermore, immunostaining of gelonin in tumor tissues also suggested expression of gelonin in the pGelonin/HPEI complexes-treated group as shown in Fig. 3B.

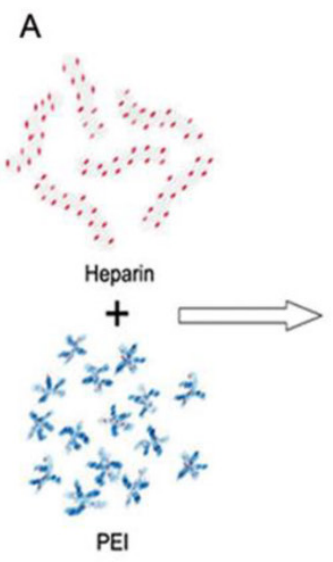

\section{B}
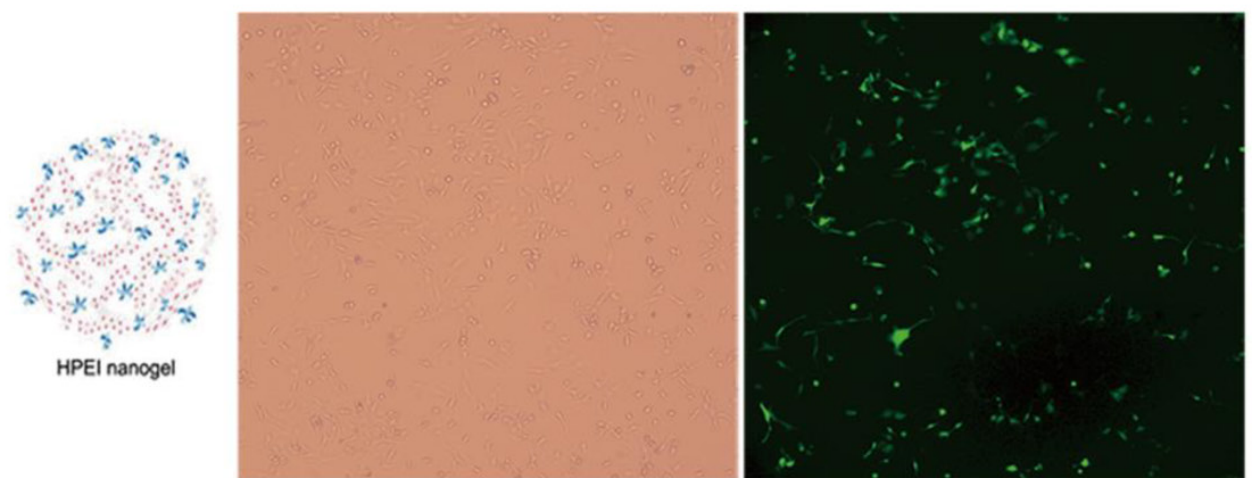

C
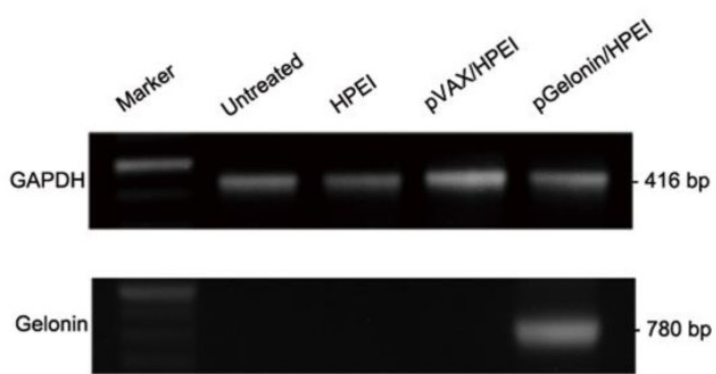

Fig. 1 Preparation and transfection efficiency of HPEI nanogels. (A) Preparation scheme of HPEI nanogels. (B) In vitro gene delivery and expression by pGFP/HPEI complexes. SKOV3 cells could be transfected by the PGFP/HPEI complexes, and a maximum expression of GFP was obtained when $2 \mu \mathrm{Pg}$ PFP was integrated with $10 \mu \mathrm{g} \mathrm{HPEI}$ nanogels. (C) Expression of gelonin in SKOV3 cells identified by RT-PCR (left) and western blot analysis (right). Gelonin mRNA and protein were detected in pGelonin/HPEl group, whereas no expression of gelonin was observed in medium alone, HPEl or pVAX/HPEI group. GAPDH served as internal control.

\section{pGelonin/HPEI complexes inhibit intraperitoneal ovarian cancer xenograft growth in nude mice}

To further evaluate the effect of pGelonin/HPEI complexes on the growth inhibition of human ovarian cancer in vivo, intraperitoneal tumors were excised and weighed at the termination of the animal experiment. The mean tumor weights of the four groups were $0.59 \pm 0.27 \mathrm{~g}, 1.10 \pm 0.23 \mathrm{~g}, 1.23 \pm 0.34 \mathrm{~g}$, and $1.43 \pm$ $0.34 \mathrm{~g}$ for pGelonin/HPEI complexes, pVAX/HPEI complexes, HPEI nanogels or medium alone, respectively ( $\mathrm{P}<0.05$, Fig. 4). The pGelonin/HPEI complexes reduced tumor weight by $45.89 \%$ compared to the pVAX/HPEI complexes. There was no significant difference in tumor weight among the control groups (P> 0.05). The characteristics of the intraperitoneal xenograft in each group were also observed. The tumor nodules were scattered predominantly in the pelvis, on the bowel surface or mesentery, and below the liver or spleen in the three control groups, whereas all of the nodules were located in the pelvis in the pGelonin/HPEI group. Two mice in the untreated group, one mouse in the HPEI group and two mice in the pVAX/HPEI group developed a small amount of epinephelus ascites. Moreover, one mice in the untreated group developed bloody ascites. No ascites were observed in the mice of the pGelonin/HPEI group. 
A

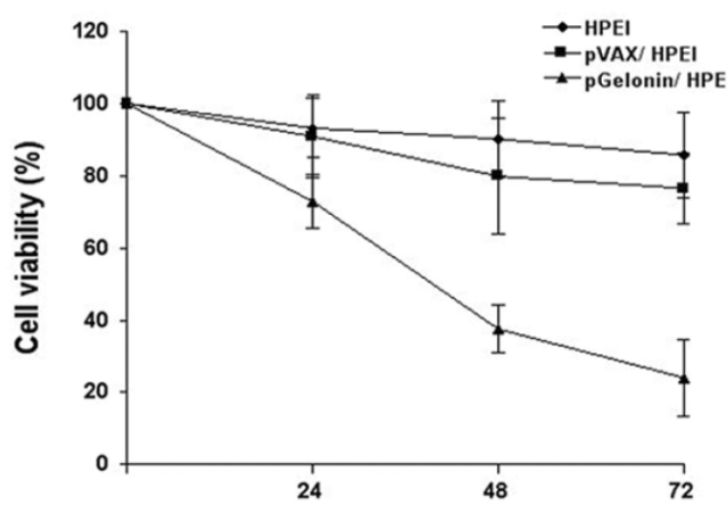

B
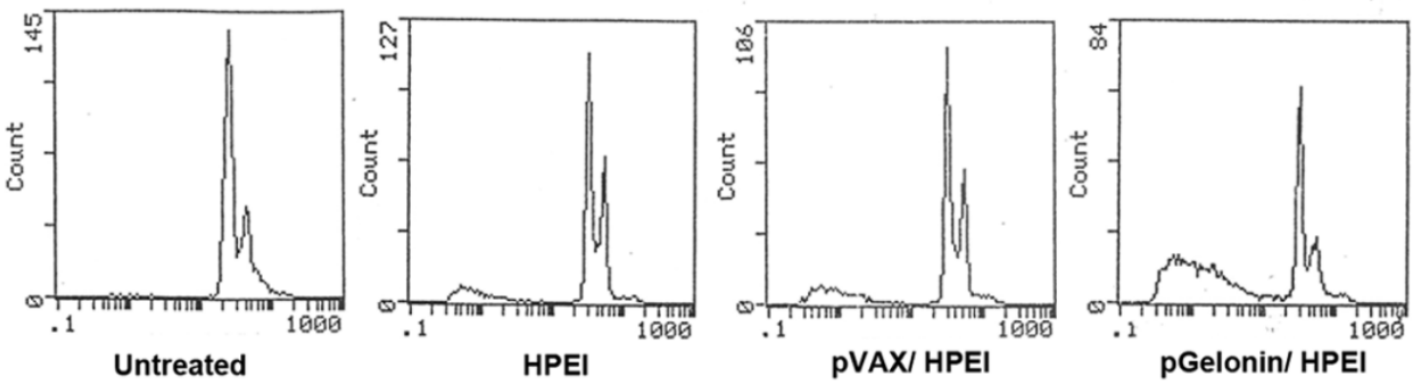

Fig. 2 Antitumor efficacy of PGFP/HPEI complexes on SKOV3 cells. (A) Inhibition of cell viability by PGFP/HPEI complexes. Cell viability was determined by MTT assay before and 24,48 and $72 \mathrm{~h}$ after transfection, respectively. Cell viability was significantly reduced in PGFP/HPEl group compared to medium alone, HPEI or PVAX/HPEl group. (B) Cellular apoptosis identified by flow cytometric analysis using propidium iodide staining method.

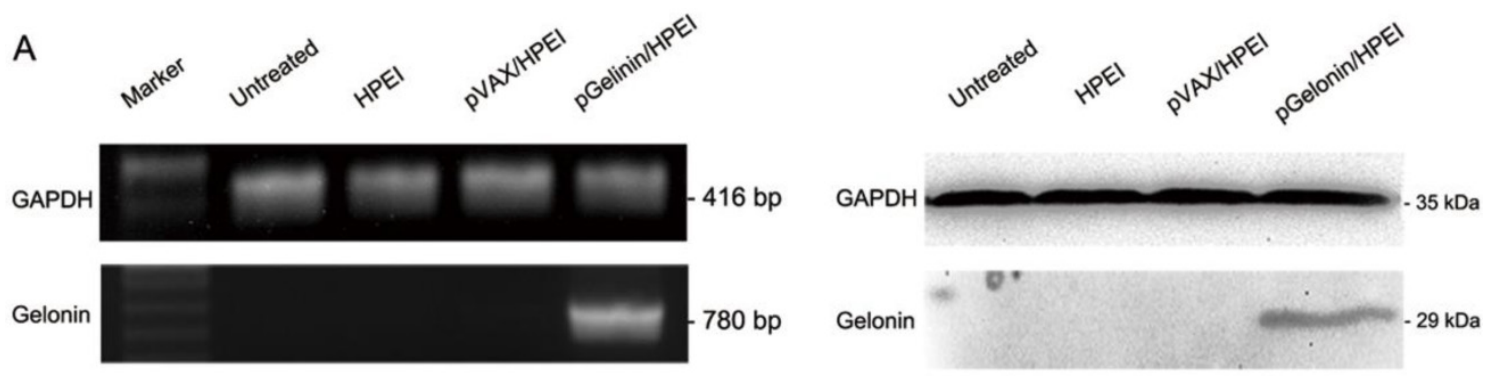

B

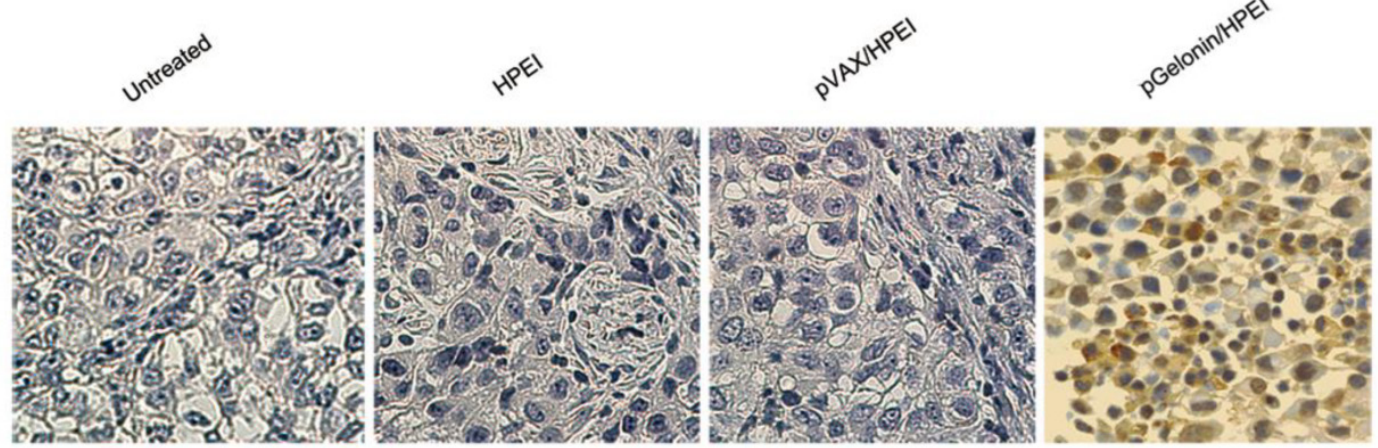

Fig. 3 Expression of gelonin in intraperitoneal tumor tissues. (A) Expression of gelonin in intraperitoneal tumor tissues detected by RT-PCR (left) and western blot analysis (right). Gelonin mRNA and protein were detected in PGelonin/HPEl group, by contrast, no expression of gelonin was observed in medium alone, HPEI or PVAX/HPEl group. GAPDH served as internal control. (B) immunostaining of gelonin in intraperitoneal tumor tissues. Original magnification, $\times 400$. 
A

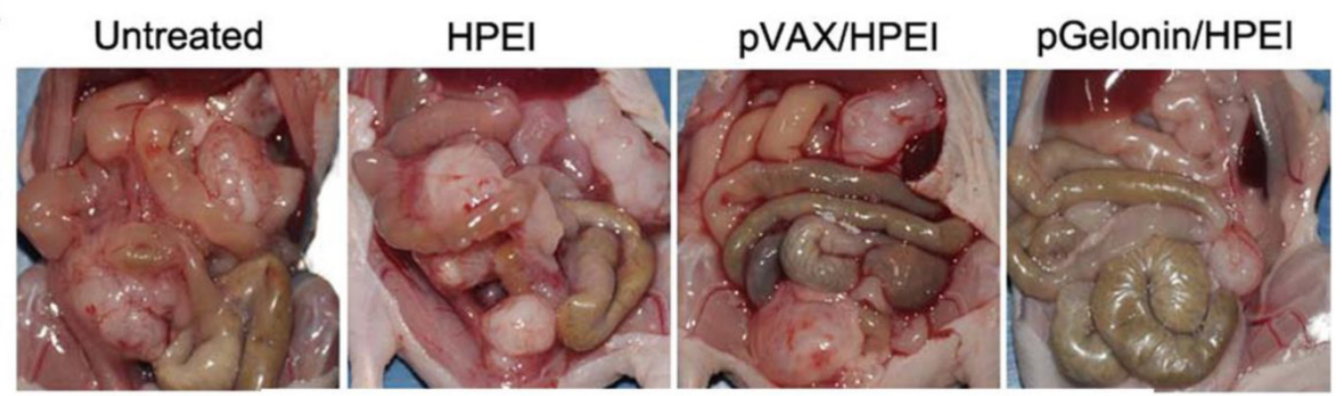

B

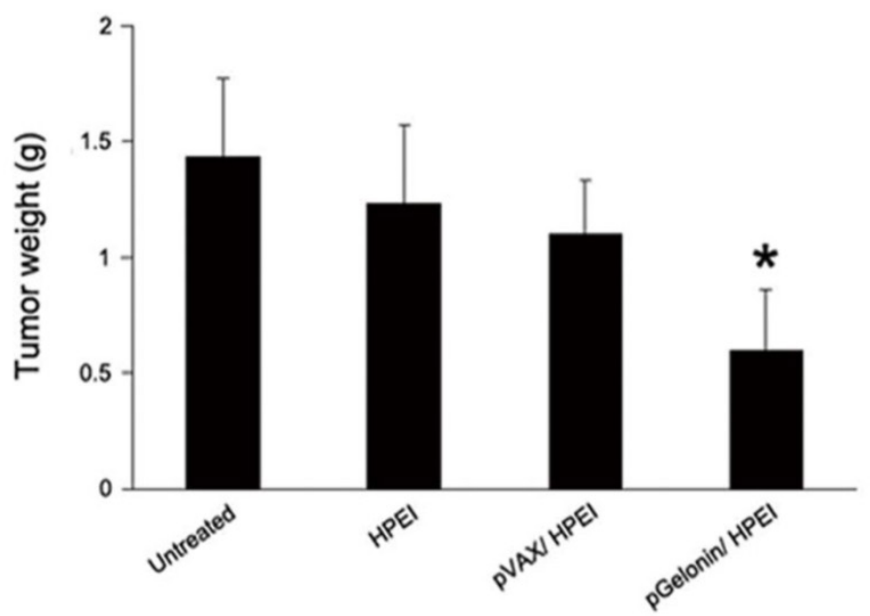

Fig. 4 pGelonin/HPEI complexes inhibited intraperitoneal tumor growth. (A) Representative macroscopic pictures of nude mice administered with $5 \%$ glucose solution, HPEI, pVAX/HPEI complexes and pGelonin/HPEI complexes, respectively. (B) Intraperitoneal tumor weight was significantly reduced in pGelonin/HPEI complexes-treated nude mice compared to the control groups. Data were expressed as the means $\pm S D, n=5$ mice/group. *, $P<0.05$.

\section{Suppression of cell proliferation and induction of cell apoptosis by pGelonin/HPEI complexes in vivo}

Ki-67 immunohistochemistry and TUNEL assay were performed to elucidate the antitumor mechanism of pGelonin/HPEI complexes in vivo. The TUNEL assay was performed out to detect apoptosis in the tumor tissues. The tumors treated with pGelonin/HPEI complexes showed a significantly greater number of TUNEL-positive nuclei than tumors in the pVAX/HPEI, HPEI or untreated groups $(22.6 \pm 5.8$ versus $10.4 \pm 4,6.2 \pm 3.4$, or $6.8 \pm 3.7(\mathrm{P}<0.05$, Fig. 5A). No significant difference in apoptosis was observed between the control groups $(\mathrm{P}>0.05)$.

To determine whether the antitumor efficacy of pGelonin/HPEI complexes correlated with decreased tumor cell proliferation, we performed Ki-67 immunohistochemistry in intraperitoneal tumor tissues. Our data showed that the percentage of Ki-67-positive cells was $15.61 \pm 3.82 \%, 47.12 \pm 8.35 \%, 44.18 \pm 5.78 \%$ and $47.79 \pm 8.84 \%$ in pGelonin/HPEI, pVAX/HPEI,
HPEI or untreated group, respectively $(\mathrm{P}<0.05$, Fig. $5 B)$.

\section{Observation of toxicity}

The animal weight was monitored every four days as shown in Fig. 6, and there were no significant differences among the four groups. No gross abnormalities were observed in the pGelonin/HPEI complexes-treated mice $(P>0.05)$. In addition, H\&E histological staining of the liver, lung, kidney, spleen and heart did not reveal any significant differences among the nude mice treated with either the pGelonin/HPEI complexes or the control therapies.

\section{Discussion}

Ovarian cancer has the highest mortality rate among reproductive cancers and currently ranks fifth in cancer deaths among women (1). Despite the improvement achieved during the last decades for ovarian cancer therapy, the 5-year survival rate remains not greater than 50\% (16). Therefore, novel agents are necessary to improve the outcomes for ovarian cancer patients. 


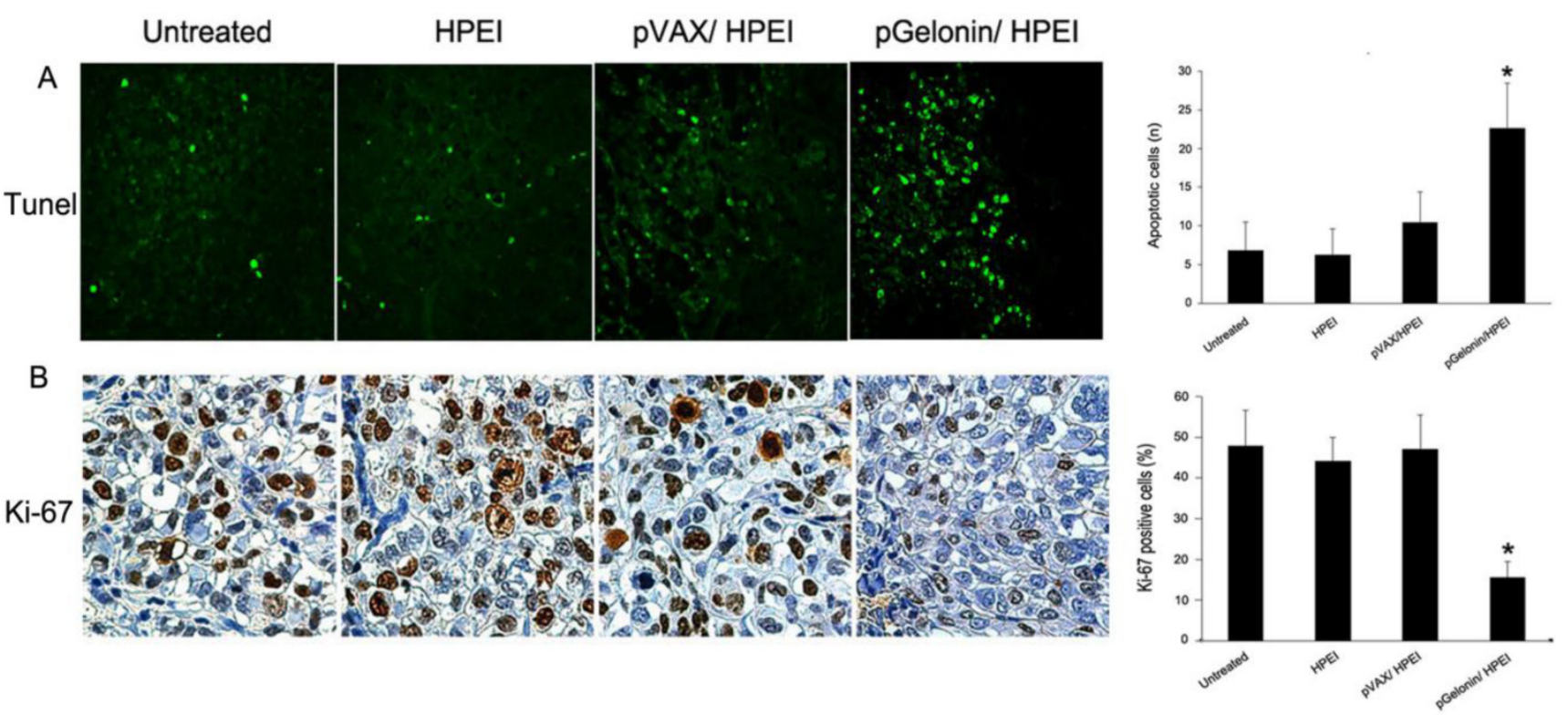

Fig. 5 TUNEL and Ki-67 immunostaining. (A) TUNEL assay showed that TUNEL-positive nuclei significantly increase in pGelonin/HPEI group compared to $5 \%$ glucose solution, HPEl or PVAX/HPEl group. *, P<0.05.×400. (B) Percentages of Ki-67-positive nuclei showed significantly decrease in pGelonin/HPEl group compared to other groups. Data were expressed as the means $\pm S D, *, P<0.05 . \times 400$.

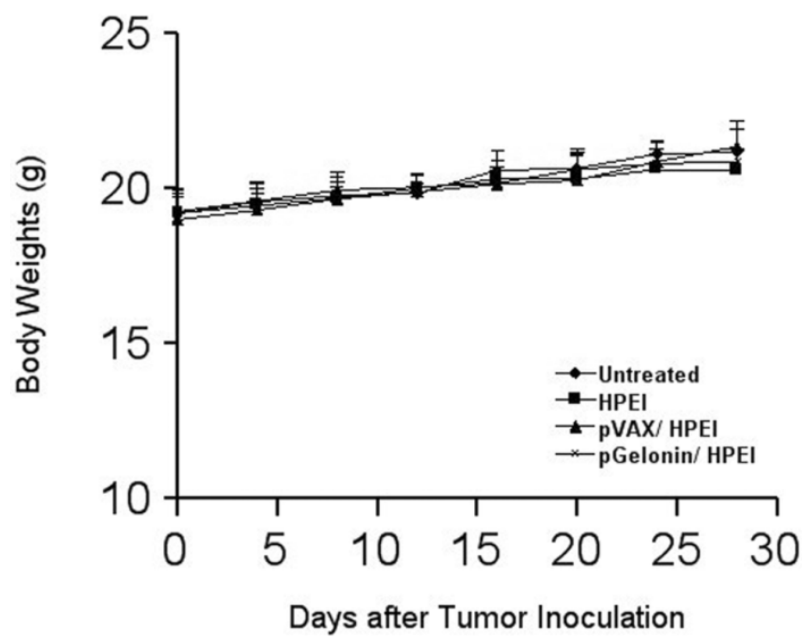

Fig. 6 Lack of toxicity-dependent weight loss in mice treated with pGelonin/HPEI complexes. There are no significant differences in weight among the four groups $(P>0.05)$.

Gelonin has received attention as a potent anti-cancer agent for its ability to cause eukaryotic cell death by removing base A4324 in $28 \mathrm{~S}$ rRNA, which prevents the association of elongation factor- 1 and -2 with the ribosomal 605 subunit (5). With different mechanisms from traditional chemotherapeutic drugs, it is a potential candidate for ovarian cancer treatment. Lacking a cell-binding domain, gelonin is nontoxic to intact cells at therapeutic doses. This characteristic has promoted the development of approaches to improve its intracellular accumulation. Previous studies showed that by directly conjugating gelonin to monoclonal antibodies, hormone or growth factor ligands, the recombinant gelonin protein inhibits the growth of several human malignant carcinomas in vitro and in vivo, including human lymphoma, melanoma, bladder carcinoma, ovarian cancer, and lung cancer $(3,17-20)$. However, its use has met with drawbacks associated with this approach, including immunogenicity, short serum half-life, drug reduction in tumor localization, protein degradation, difficulties of refinement and high cost.

During the past decade, molecular chemotherapy has been widely studied with the purpose of eradicating cancer cells via a genetically expressed toxin. Previous studies tested the antitumor potential of a recombinant plasmid DNA expressing diphtheria toxin A-fragment in several ovarian cancer cells and in a heterotopic ovarian cancer model. Successful expression of diphtheria toxin A-fragment and the reduction of ovarian cancer growth were observed (21). Therefore, in this study, attention was focused on a plasmid DNA vector-based strategy. The gelonin gene was cloned into the pVAX1 plasmid vector and then transfected into SKOV3 ovarian cancer cells. The antitumor activity was investigated in vitro and in vivo.

Due to recent advances in gene transfer technology, gene therapy has become a reality with some patients currently undergoing clinical gene therapy trials. However, the science of efficient and safe gene transfer technology remains in its infancy and is a major challenge for clinical application. Currently, both nonviral and viral vectors have been used in gene transfer. Because of the adverse side effects caused by viral vectors, a novel nonviral gene delivery system based on PEI was used. PEI is one of the 
most efficient nonviral vectors; however, its clinical application is restricted because of its unbiodegradability and high cytotoxicity. Therefore, modifications to PEI have been attempted, and several PEI-based delivery systems have been used in gene therapy (22-27). In our laboratory, PEI was chemically conjugated with heparin to transform its properties. These novel biodegradable cationic HPEI nanogels have been used to deliver several functional genes, such as VSVMP, FILIP1L, IL-15 and mice surviving-T34A, to different cancer cells, with low cytotoxicity and high transfection efficiency as previously described (11-14). In this study, the transfection efficiency of HPEI nanogels was evaluated in SKOV3 cells using plasmid GFP. The maximum transfection efficiency was obtained when $2 \mu \mathrm{g}$ pGFP was integrated with $10 \mu \mathrm{g}$ HPEI nanogels. As shown in fig.1B, there are numerous GFP-positive cells, indicating that HPEI nanogels efficiently transfect plasmid DNA into SKOV3 cells. Therefore, we used HPEI nanogels as the delivery system to assess the antitumor effect of pGelonin, as well as the transfection efficacy and therapeutic safety. To the best of our knowledge, this is the first study in which gelonin toxin is introduced into SKOV3 ovarian cancer cells using recombinant plasmid DNA and biodegradable cationic HPEI nanogels. The efficient delivery of pGelonin incorporated in HPEI nanogels was confirmed by the successful expression of gelonin in vitro and in vivo.

In the current study, pGelonin/HPEI complexes were transfected into SKOV3 human ovarian cancer cells and administered to nude mice bearing intraperitoneal ovarian carcinomatosis. Initially, we examined the transfection efficiency of the HPEI nanogels. The RT-PCR and western blot analysis results showed that gelonin was successfully expressed in the pGelonin/HPEI group both in cell culture and in the intraperitoneal tumor tissues. Subsequently, we tested the antitumor activity of pGelonin/HPEI complexes in vitro. The MTT assay indicated that pGelonin/HPEI complexes significantly inhibited the viability of SKOV3 cancer cells, whereas the pVAX/HPEI complexes and HPEI nanogels did not significantly inhibit cell viability. Flow cytometric analysis revealed that there were more apoptotic cells in the pGelonin/HPEI complex group compared to the control groups. Our data indicated that pGelonin effectively inhibited the growth of SKOV3 ovarian cancer cells by reducing cell viability and inducing apoptosis in vitro. Based on the data, we investigated the antitumor efficiency of pGelonin in vivo. Nude mice bearing intraperitoneal xenografts were administered i.p. injections of pGelonin/HPEI complexes. The results suggest that pGelonin/HPEI complexes significantly suppress intraperitoneal tumor growth. The tumor weight in the pGelonin/HPEI-treated mice was lower compared to the control groups $(\mathrm{P}<0.05)$. This study supports the hypothesis that pGelonin possesses antitumor activity against ovarian cancer in vivo. Furthermore, cell apoptosis and proliferation in intraperitoneal tumor tissues were evaluated using TUNEL assay and Ki-67 immunohistochemistry. Increased apoptosis and inhibition of cell proliferation were observed in tumor tissue sections in the pGelonin/HPEI group compared to the control groups $(\mathrm{P}<0.05)$. These results indicate that the antitumor efficacy of pGelonin against intraperitoneal carcinomatosis may be partially due to the induction of apoptosis and inhibition of cell proliferation. We also evaluated the safety of our treatment. No gross abnormalities were observed in the pGelonin/HPEI complexes-treated mice. In addition, H\&E histological staining of the main organ tissues, such as the heart, liver, spleen, lung and kidney, did not reveal any significant differences among nude mice treated with either pGelonin/HPEI complexes or the control therapies.

Our data indicate that treatment with the pGelonin/HPEI complexes is safe and without obvious toxicity at a low dosage $(5 \mu \mathrm{g}$ DNA/ $25 \mu \mathrm{g}$ HPEI per mouse) and with i.p. administration. However, the application of HPEI nanogels as a gene delivery system requires further investigation, and molecular chemotherapy using a plasmid expressing gelonin toxin in other cancers may be required, and further studies are paramount. Because of the potent inhibition of protein synthesis following transfection and expression of the plasmid expressing gelonin, it is possible that cancer cells will not exhibit resistance to this novel therapeutic approach using pGelonin/HPEI complexes. With different antitumor mechanisms from traditional chemotherapy, HPEI nanogel-delivered pGelonin may have potential application for ovarian cancer treatment.

In conclusion, our results suggest that HPEI nanogels effectively transfect pGelonin into SKOV3 human ovarian cancer cells and that successful expression of the gene encoding the gelonin toxin resulted in efficient inhibition of intraperitoneal ovarian cancer growth through the induction of apoptosis and inhibition of cell proliferation. The present findings provide evidence that HPEI nanogel-delivered pGelonin may have a potentially promising application against ovarian cancer.

\section{Acknowledgments}

This work was financially supported by the National 973 Program of China (2010CB529905, 2011CB910703), the National Natural Science Foundation of China (NSFC81071861) and the National 


\section{Science and Technology Major Project (2009zx09503-020).}

\section{Competing Interests}

The authors have declared that no competing interests exist.

\section{References}

1. Siegel R, Naishadham D, and Jemal A. Cancer statistics, 2012. CA Cancer J Clin. 2012;62: 10-29.

2. Stirpe F, Olsnes SE and Pihl A. Gelonin, a new inhibitor of protein synthesis, nontoxic to intact cells. J Biol Chem. 1980;255: 6947-53.

3. Talpaz M, Kantarjian HM, Freireich EJ, et al. Phase I clinical trial of the anti-CD33 immunotoxin HuM195/rGel. American Association for Cancer Research; Abstract R5362, 94th Annual Meeting. 2003;44: 1066.

4. Gawlak SL, Neubauer M, Klei HE, et al. Molecular, bioligical, and preliminary structural analysis of recombinant bryodin 1, a ribosome-inactivating protein from the plant bryonia dioica. Biochemistry. 1997;36:3095-103.

5. Kreitman RJ. Immunotoxins for targeted cancer therapy. Aaps J. 2006;8: E532-51.

6. Raki M, Rein DT, Kanerva A, et al. Gene transfer approaches for gynecological diseases. Mol Ther. 2006;14: 154-63.

7. Boussif $\mathrm{O}$, Lezoualch F, Zanta MA, et al. A versatile vector for gene and oligonucleotide transfer into cells in culture and in vivo: Polyethyleneimine. Proc Natl Acad Sci USA. 1995;92: 7297-301.

8. Lungwitz U, Breunig M, Blunk T, et al. Polyethyleneimine-based non-viral gene delivery systems. Eur J Pharm Biopharm. 2005;60: 247-66.

9. Godbey WT, Wu KK, Mikos AG. Size Matters: molecular weight affects the efficiency of poly(ethyleneimine) as a gene delivery vehicle. J Biomed Mater Res. 1999;45: 268-275.

10. Kunath K, von Harpe A, Fischer D, et al. Low-molecular-weight polyethyleneimine as a non-viral vector for DNA delivery: comparison of physicochemical properties, transfection efficiency and in vivo distribution with high-molecular-weight polyethyleneimine. J Controlled Release. 2003;89: 113-125.

11. Gou M, Men K, Zhang J, et al. Efficient inhibition of C-26 colon carcinoma by VSVMP gene delivered by biodegradable cationic nanogel derived from polyethyleneimine. ACS Nano. 2010;4: 5573-84.

12. Xie C, Gou M, Yi T, et al. Efficient inhibition of ovarian cancer by truncation mutant of FILIP1L gene delivered by novel biodegradable cationic heparin-polyethyleneimine nanogels. Human Gene Ther. 2011;22: 1413-22.

13. Liu P, Gou M, Yi T, et al. Efficient inhibition of an intraperitoneal xenograft model of human ovarian cancer by HSulf- 1 gene delivered by biodegradable cationic heparin-polyethyleneimine nanogels. Oncol Rep. 2012;27: 363-70.

14. Zhang L, Gao X, Men K, et al. Gene therapy for C-26 colon cancer using heparin-polyethyleneimine nanoparticle-mediated survivin T34A. Int J Nanomedicine. 2011;6: 2419-27.

15. Lin $\mathrm{XJ}$, Chen $\mathrm{XC}$, Wang $\mathrm{L}$, et al. Dynamic progression of an intraperitoneal xenograft model of human ovarian cancer and its potential for preclinical trials. J Exp Clin Cancer Res. 2007;26: 467- 74

16. DiSaia PJ, Creasman WT. Epithelial ovarian cancer. In: Clinical Gynecologic Oncology. 5th edition. DiSaia PJ and Creasman WT (eds). Mosby, St. Louis, 1997.

17. Veenendaal LM, Jin H, Ran S, et al. In vitro and in vivo studies of a VEGF121/rGelonin chimeric fusion toxin targeting the neovasculature of solid tumors. Proc Natl Acad Sci USA. 2002;99: 7866-71.

18. Rosenblum MG, Shawver LK, Marks JW, et al. Recombinant immunotoxins directed against the c-erb-2/HER2/neu oncogene product: in vitro cytotoxicity, pharmacokinetics, and in vivo efficacy studies in xenograft models. Clin Cancer Res. 1999;5: 865-74.

19. Duzkale H, Pagliaro LC, Rosenblum MG, et al. Bone marrow purging studies in acute myelogenous leukemia using the recombinant anti-CD33 immunotoxin HuM195/rGel. Biol Blood Marrow Transplant 2003;9: 36-72.

20. Singh V, Sairam MR, Bhargavi GN, et al. Hormonotoxins. Preparation and characterization of ovine luteinizing hormone-gelonin conjugate. J biological chemistry. 1989;264: 3089-95.

21. Mizrahi A, Hochberg A, Amiur S, et al. Targeting diphtheria toxin and TNF alpha expression in ovarian tumors using the H19 regulatory sequences. Int J Clin Exp Med. 2010;3: 270-82.

22. Breunig M, Lungwitz U, Liebl R, et al. Breaking up the correlation between efficacy and toxicity for nonviral gene delivery. Proc Natl Acad Sci USA. 2007; 104: 14454-9.

23. Alexis F, Lo SL, Wang S. Covalent attachment of low molecular weight poly(ethylene imine) improves tat peptide mediated gene delivery. Adv Mater. 2006;18: 2174-8.

24. Wen Y, Pan S, Luo X, et al. Biodegradable low molecular weight polyethyleneimine derivative as low toxicity and efficient gene vector. Bioconjugate Chem. 2009;20: 322-32.

25. Kim YH, Park JH, Lee M, et al. Polyethyleneimine with acid-labile linkages as a biodegradable gene carrier. J Controlled Release. 2005;103:209-19.
26. Park MR, Kim HW, Hwang CS, et al. Highly efficient gene transfer with degradable poly (ester amine) based on poly (ethylene glycol) diacrylate and polyethyleneimine in vitro and in vivo. J Gene Med. 2008;10: 198-207.

27. Gosselin MA, Guo W, Lee RJ. Efficient gene transfer using reversibly cross-linked low molecular weight polyethyleneimine. Bioconjugate Chem. 2001;12: 989-94. 